\title{
The design and operation of an improved olfactometer for behavioral and physiological investigation'
}

\author{
HERBERT STONE, ${ }^{2}$ GORDON PRYOR, and GERALD \\ STEINMETZ, STANFORD RESEARCH INSTITUTE, Menlo \\ Park, California 94025
}

An improved olfactometer is described, and its range of application for the study of the olfaction in man is discussed. The apparatus is based on air dilution; an odorant is diluted in a constant-flowing stream of air. The odor-containing air flows to $S$, who is seated in an enclosed partition with his head in a Plexiglas hood. A visual signal system allows communication between $E$ and $S$. Precision flowmeters, solenoid valves, one-way floats, and an automated control system permit $E$ to select rapidly a large range of stimuli, stimulus concentrations, and trial lengths. The apparatus has been used in a variety of situations ranging from absolute threshold detection to adaptation and recovery.

Quantitative behavioral and physiological investigations of the olfactory responses of man have always been difficult because of the many problems associated with odor presentation. Most techniques have utilized a dilution system, the dilutent being air or some liquid solvent assumed not to alter the physicochemical characteristics of the stimulus (Stone, 1963a). Air dilution is accepted generally as the most reliable method and involves introducing a known quantity of an odorous chemical into a stream of air, usually flowing in a confined system at a specified flow rate. The idea of an olfactometer based on the principle of air dilution was first proposed by Zwaardemaker (1921) and has since been utilized by several investigators.

Another important difference between the various systems is the method of stimulus delivery to the olfactory region-usually accomplished by a $\mathrm{S}$ sniffing the air stream on cue, or by injection into his nose. The injection method, also known as the Elsberg technique (Elsberg \& Levy, 1935), has an apparent advantage of simplicity and avoidance of individual variations in sniff volume, but several observers have also noted its difficulties. Jones (1953) reported that when odor-containing air is forced (blasted) into the nose, responses were influenced by the aerodynamics of the nose. Wenzel (1955) emphasized the need for a naturally breathing or sniffing $S$ to yield more reliable results. These and other experimenters have raised doubts about the validity of data resulting from use of the blast injection technique, since perception of odor strength appeared to be more directly related to injection pressure than to stimulus concentration.

The literature on olfactometric equipment has been reviewed in detail (see Stone, Pangborn, \& Ough, 1965; Amerine et al, 1965 ) and the following have been suggested as criteria for a good system: (a) continuous flow of dilutent air; (b) flexibility in selection of the stimulus and stimulus concentration; (c) rapid introduction and removal of the stimulus; (d) naturally breathing Ss; (e) ability to present more than one stimulus at a time; (f) ease of operation, data recording, and cleaning; (g) requirement of less than $5 \mathrm{cc}$ of a stimulus; and (h) minimal cost to construct and maintain.

The olfactometric system described in this report incorporates the above suggestions. It is an "improved" model of the air-dilution unit used by Stone for several olfactory investigations

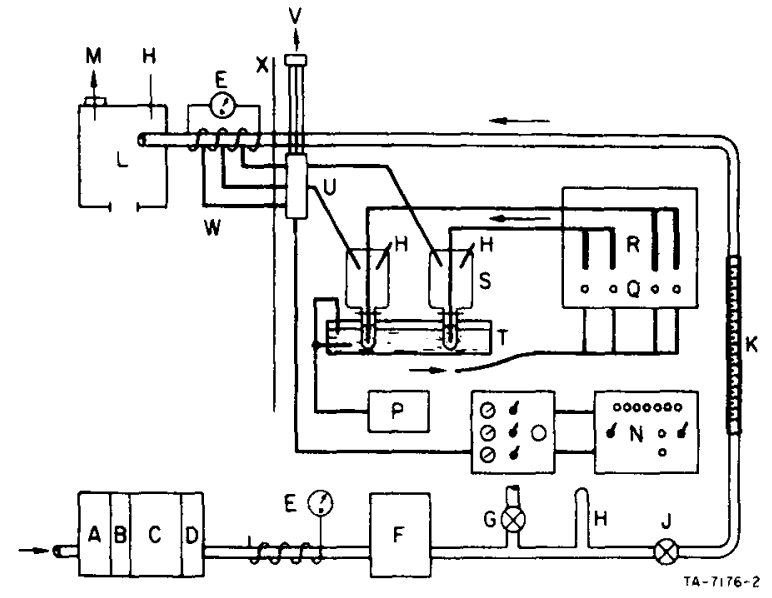

Fig. 1. Schematic diagram of the olfactometer: $A$, high-speed, squirrel-cage blower; B, coarse filter; C, refrigeration unit; D, high speed, motor-operated blower; E, heating element, Variaccontrolled; F, activated charcoal filters (Dorex, Type H, No. 42 from the W. B. Connor Engineering Corp., Danbury, Conn.); G, by-pass valve; $H$, thermometers; J, stopcock for air-flow regulation $(75-\mathrm{mm}$ bore); $\mathrm{K}$, air-flow meter (precision bore, regulator tube, Fischer-Porter No. B5-27,10/27); L, Plexiglas hood (wall thickness, $10 \mathrm{~mm}$; height, $30 \mathrm{~cm}$; other dimensions, $38 \mathrm{~cm}$ ); $M$, exhaust motor vented to outside; $N$, main control panel with signal lights for recording responses; $\mathbf{O}$, electronic control panel (see Fig. 3); P, temperature recorders and regulators; $Q$, regulator needle valves (Whitey Valves, Model No. 22RS4-A); R, flowmeters (Matheson Co., Inc., Nos. 600, 601); S, diffusion bulbs (see Fig. 2); T, water bath; U, solenoid valves (Automatic Switch Co., Model No. 832043V; gas/liquid, 1/8-in. pipe, stainless steel, $110 \mathrm{Vac}$ ); $\mathrm{V}$, back-pressure regulator (water level adjusted to main air-line back pressure); W, one-way floats (see Fig. 2); $X$, partition separating $E$ from $S$.

in man (Ough \& Stone, 1961; Stone \& Bosley, 1965). Most of the suggested features were met in the early design of the apparatus. However, improvements in regulators, flowmeters, one-way floats, and automated switching devices have led to a significantly improved system that allows increased flexibility and precision. Rapid and precise presentation of stimuli, with automated timing control, is now possible and repeated trials on the same $\mathrm{S}$ can be conducted in sessions lasting as long as $2 \mathrm{~h}$.

\section{Design Characteristics}

A schematic diagram of the complete system is shown in Fig. 1. Figures 2 and 3 depict the one-way glass float, the odor diffusion bulb, and the circuit diagram for the timing and switching systems.

\section{Operation}

Briefly, the function and operation of the unit (see Fig. 1) are as follows. Fresh outdoor air is pulled into the system at A, filtered, cooled (to remove solubles), and speeded at B, C, and D. It is heated to any desired temperature at $\mathrm{E}$ (usually $25 \mathrm{deg} \mathrm{C}$ ) 


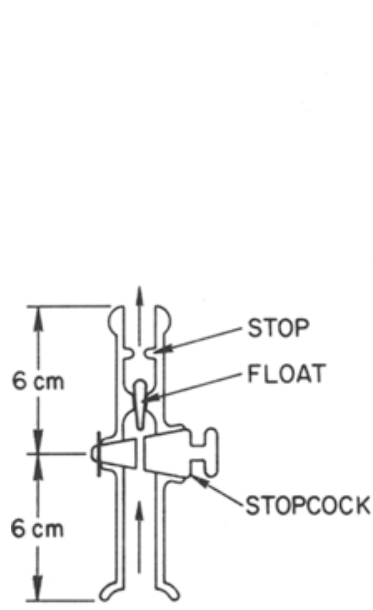

I

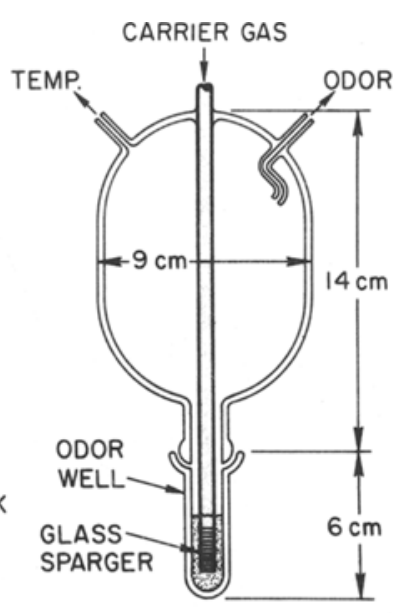

TA-7176-1

Fig. 2. Schematic diagrams of the one-way float (I) and the odor diffusion bulb (II). The one-way float is made from 8-mm-bore tubing and a No. 4 stopcock. The overall length is $12 \mathrm{~cm}$. Ball joints at either end are held in the unit with No. 18 C-clamps. That portion of the float that touches the wall is ground, as is the wall itself, thereby ensuring a tight fit. The diffusion bulb may be prepared from a 250 -cc (or smaller), round-bottom flask, overall dimensions $10 \times 7 \mathrm{~cm}$, with $8-\mathrm{mm}$ bore tubing for the connections. The odor well is $5 \mathrm{~cm}$ long and held in position with a No. 35 C-clamp. The extra-coarse scintered glass sparger can be obtained from any chemical glassware supply house. Cleaning is accomplished by rinsing in freshly prepared alcoholic KOH and dichromate followed by distilled water, and drying overnight in an oven at $100 \mathrm{deg} C$.

and refiltered at F. Airflow rate is controlled by Stopcock I, and measured at Flowmeter K. Flow rates (odor dilution factor) of from 185 to $325 \mathrm{l} / \mathrm{min}$ are obtained. The air, now "purified" and of constant temperatures and flow rate, passes from $\mathrm{K}$ directly to the S's Plexiglas hood (L). The experimenter, seated in front of the controls at $\mathrm{N}, \mathrm{O}, \mathrm{Q}$, and $\mathrm{R}$, meters the carrier gas (purified, water-pumped $\mathbf{N}_{2}$ ) through one of the flowmeters $(R)$ into the diffusion bulb (S), where saturation is achieved. This saturated vapor is vented through the solenoid $(U)$ to the outside by way of the back pressure regulator (V). It reaches the $S$ once the solenoid is activated by passing through the one-way float $(W)$. Dilution with the main air stream occurs at the heated mixing chamber (E). The circuitry of Control Panel O (see Fig. 3) is designed to provide maximum flexibility. To present stimuli under automated control, the investigator presets timers controlling trial length and intertrial interval, and solenoid valves are activated automatically. Changes in stimulus strength are made during intertrial intervals. Each solenoid has manual override switches that separate timers from the control circuit and enable $E$ to present stimuli manually. Control Panel N provides $E$ with S's response information in the form of light signals for odor detection (or nondetection) and odor strength estimation. It also includes switches that control light cues on S's panel indicating "test trial" and "no trial." Again, these may be shifted to E's manual control.

\section{Application}

The equipment has been used in this laboratory primarily for the investigation of olfactory adaptation-recovery phenomena and for threshold measurement. To date, three techniques have been satisfactorily employed:

(1) Absolute thresholds have been quickly and accurately determined with a single timer-controlled solenoid in the line between a given odorant-containing diffusion bulb and the main air stream. E presets a stimulus strength level at the flowmeter $(Q$ in Fig. 1) and presents it for a preset period via the timed solenoid circuit by closing a switch and starting the cycle. During the intertrial interval, which is also preset and controlled by the delay timer, he receives and records S's response and presets the stimulus strength for the next trial of the cycle. To present an air blank "stimulus," E closes the flowmeter but cycles the timers and solenoid as before so that $S$ receives no differential auditory cue relative to odor or absence of odor. Because of the water back-pressure provision on the solenoid vent, the changes in stimulus strength are abrupt rather than gradual. This permits the use of short stimulus periods with the assurance that $S$ receives full stimulus strength for the trial period without overlap into the intertrial interval. Thus, adaptation effects are minimized in a series of threshold trials and more accurate data are obtained. Currently, a 4-sec stimulus period and a $20-\mathrm{sec}$ intertrial interval are employed. Without the automated equipment such a regimen would severely strain, if not overtax, E's capacity to set manually stimulus strengths and time intervals. Timing accuracy, of course, far exceeds that obtained when settings are changed with stopwatch cues.

(2) Adaptation as a function of S's time in the presence of an adapting stimulus of given strength is also measured quickly and accurately. Using the same odorant in each of two diffusion bulbs, the timer-controlled solenoid line from one bulb is used to present an adapting stimulus, and the timer-controlled solenoid line from the other is used to present the test stimulus. The investigator records changes in the threshold as a function of adaptation and time (i.e., a preset adapting stimulus can be presented via one solenoid line for a preset period). At the end of the period, the adapting stimulus is vented while the test stimulus

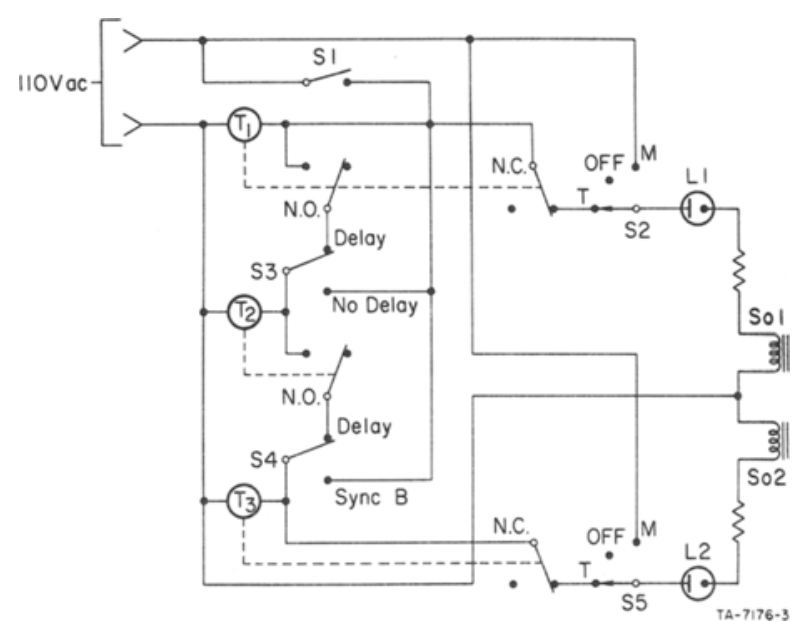

Fig. 3. Circuit diagram for the electronic timing system: So 1 , solenoid in one odor line between diffusion bulb and main air stream; So2, solenoid in a second odor line; $L 1$ and L2, lights controlled by Sol and So2, respectively; T1, timer control for Sol; T2, timer control of optional delay period between $\mathrm{T} 1$ and T3; S1, main on/off switch; S2, off/on switch for timercontrolled circuits; S3, manual override for Sol (by-pass timer circuit and switch into timer control or manual on/off); S4, permits timer delay to start simultaneously with $T 1$ or at completion of $\mathrm{T} 1$; S5, synchronous delay for $\mathrm{T} 3$ which permits T3 to be controlled by $\mathrm{T} 2$ or to start simultaneously with $\mathrm{T} 1 ; \mathbf{S b}$, manual override for So2, same as S3; M, manual; T, timed; N.C., normally closed. 
is presented. Again, the test period is kept short to minimize interference with the adaptation process; some adaptation does occur. ${ }^{3}$ The back-pressure provision prevents lag and/or carryover between the adapting and test stimuli. The E need only "chase" and record the rising threshold by changing successive threshold test strengths during the adaptation period. Over several sessions, an adaptation curve is developed for each S. Since adaptation is most rapid initially, the threshold change may be tested more frequently at the beginning of a session than toward the end of adaptation. This can be done by changing the timer setting controlling the solenoid on the adapting stimulus line.

(3) In the measurement of recovery after adaptation, the procedure is similar except that the adapting stimulus is zero (deleted from circuit) and very brief threshold test trials are presented. These are presented more frequently during the initial, rapid phase of recovery rather than toward the end.

The equipment may be expected to function equally well in studies of cross-adaptation, odor mixtures, and descriptive analysis. At this time, the two principal values of the apparatus lie in the versatility of the controlling circuitry and in the precision of control of stimulus strengths and duration of test periods and intertrial intervals.

\section{DISCUSSION}

The changes reported here from the earlier version of the olfactometer have resulted in a significant improvement in the use of the instrument and in obtaining more reliable behavioral information from the Ss. For example, it was observed in some earlier experiments that the flow of the main air stream $(\geqslant 250 \mathrm{l} / \mathrm{min}$ ) would create a slight negative pressure at the odor inlet to the air stream (Venturi effect), thereby "pulling" odorant molecules into the main air stream during intertrial, no-odor intervals. The one-way glass float eliminates this problem.

The solenoid switches vastly improve stimulus presentation. The E can turn on the stimulus control valve at the desired flow and then, by flipping a switch, activate the solenoid enabling the odorant to flow to $\mathrm{S}$. The timer keeps the solenoid open for the preset time and then closes it for the intertrial interval. At this time $E$ can shut off the stimulus or change to another setting (i.e., increase or decrease stimulus concentration). The stimulus passing into the air stream is vented outdoors. It was also found necessary to have a back pressure on the solenoid vent equal to that at the one-way float in order to equilibrate the entire system. This was accomplished with a gas trap calibrated with water to a specific level. Prior to providing for back pressure, stimuli lagged in arrival at $S$ 's hood as dead space within the valve was filled.

The location of the air-odor mixing unit (with three inlets) was moved within $6.8 \mathrm{in}$. of $S$ for several reasons. When the mixing unit was more than a few feet from the odor hood, there was a possibility that some odorant molecules might be adsorbing on, and desorbing from, the glass and providing misleading information (see Stone, 1968). Also, as mixing distance from the $S$ is decreased, time lag of stimulus arrival is decreased. Moving this unit within $6 \mathrm{in}$. of the odor hood was found to be sufficient for mixing. It also reduced the likelihood of adsorption and decreased stimulus travel time to zero. To further minimize the adsorption-desorption problem, the mixing unit was made of quartz glass with an inner silicone coating and a thin Teflon sleeve around the last 4 in. to the hood. Finally, the mixing unit was heated to keep the inner surface warm and thus further reduce the possibility of adsorption.

Sufficient experiments have been conducted with an earlier version of this olfactometer to demonstrate its usefulness in the study of olfaction in man (Ough \& Stone, 1961; Stone, 1963a, Stone \& Bosley, 1965; Stone et al, 1967). Where appropriate, quantitative recovery of the stimulus was achieved to verify concentrations as perceived by S (Stone, 1963b). ${ }^{4}$ By knowing the main air and carrier-gas flow rates, the molecular weight of the stimulus, and the vapor pressure at the test temperature, the concentration (in $\mathrm{g} / \mathrm{l}$ ) of odorant entering the Plexiglas hood can be calculated. To determine concentration at the olfactory region, sniff volume (average $400-600 \mathrm{ml}$ ) and Stuiver's correction for loss $(98 \%)$ can be applied to the calculated values (Stuiver, 1958).

The olfactometer is usually operated at an air-flow rate of $250 \mathrm{l} / \mathrm{min}$ although we have raised (to $3251 / \mathrm{min}$ ) or lowered (to $180 \mathrm{l} / \mathrm{min}$ ) the rate in order to achieve a greater stimulus concentration range. Using various flow rates, it has been found that $S$ sensitivity is independent of the change in flow (see Table 1). Ss do not report that these flows are too strong; approximately $0.5 \mathrm{~cm}$ from the edge of the air inlet tube is a perforated Teflon plate that breaks the velocity of the incoming air.

The question of what constitutes a suitable air supply for olfaction experiments may not be critical provided suitable precautions have been taken. It has been our experience that the combination of a coarse filter, precooling, and activated charcoal filters will do an excellent job of reducing problems of contaminated air. Piped, compressed air in most laboratories contains a fine oil mist that will pass through most filters (and through most low-temperature traps). Tanks of compressed air can be purchased and these usually are oil-free; however, air-flow rates would have to be reduced to keep the cost reasonable. Reducing the air-flow rate to less than $1501 / \mathrm{min}$ would reduce the range of odor dilution possible and would necessitate some changes in the equipment, e.g., smaller bore tubing. Ideally, one would like to have "pure" air; however, this may require such expensive and elaborate equipment that its cost may far exceed its value. Finally, results obtained with this equipment are consistent over several years, and specific threshold values are in good agreement with results reported elsewhere. Since the literature contains no threshold data derived from studies using "pure" air, there is no reason to believe that our technique using ambient filtered air is inadequate.

A second part of the system that warrants discussion is the use of flowmeters instead of capillary tubes. A capillary represents the most accurate method for presenting a specified amount of carrier gas (and odor). The accuracy of the flowmeters (manufacturer states $1 \%$ of full scale) is entirely dependent on E's ability to read the float setting (which is at the middle of the float) and to reproduce these settings with some degree of accuracy. We routinely calibrate the flowmeters during the course of an experiment and find good agreement with our calibration curves (those supplied by the manufacturer are usually inadequate). In our opinion, the flowmeters are more advantageous than the capillaries because the former are more flexible and only one regulator need be manipulated. Since capillaries have only one flow, E must have a sufficient number of them to be able to present a wide range of stimuli concentrations. The range of olfactory responses in man is not nearly as great as that of other sense modalities, and two $\log$ units usually are sufficient to go from "just detectable" to "extremely intense." Thus, it is necessary to have smaller concentration steps in order to obtain usable data. Capillaries also require considerably more glassware which, in our experience, increases equipment and maintenance costs.

The five-sided Plexiglas hood $(30 \mathrm{~cm}$ high $\times 38 \mathrm{~cm}$ wide $\mathrm{x}$ $38 \mathrm{~cm}$ deep, with no bottom) used as the "enclosed" environment, within which $S$ keeps his head during testing, has proved to be quite satisfactory. Not only do Ss not report feelings of confinement, but the signal system used for communication between $E$ and $S$ can be placed in an accessible position. The possibility that the Plexiglas contributes odor or adsorbs (and 
Table 1

The Effect of Changing Air Flow on Absolute Odor Detection Thresholds for Methyl Isobutyl Ketone

\begin{tabular}{ccc}
\hline Subject & $\begin{array}{c}\text { Air Flow } \\
(1 \mathrm{~min})\end{array}$ & $\begin{array}{c}\text { Threshold Conc. } \\
\mathrm{mg} / 1\end{array}$ \\
\hline 1 & 247.0 & .0010 \\
& 326.0 & .0010 \\
2 & 247.0 & .0009 \\
& 293.3 & .0009 \\
& 326.0 & .0008 \\
\hline
\end{tabular}

The thresholds were determined using a modified tracking procedure (Stone et al, 1967); $S$ received 50 trials in a session in which half of the presentations were blanks and half were stimuli. Similar results were obtained with several other Ss.

desorbs) any of the test stimuli was given careful consideration. Attempts to demonstrate this (by chemical means) were not successful, primarily because of the nonstatic nature of the system; i.e., the air was always flowing at a rate greater than $200 \mathrm{l} / \mathrm{min}$ (a complete changeover of the space occurred in less than $2 \mathrm{sec}$ ). As a precautionary measure, after each test session the walls are rinsed with acetone and warm distilled water, then wiped dry.

\section{REFERENCES}

AMERINE, M. A., PANGBORN, R. M., \& ROESSLER, E. B. Principles of sensory evaluation of food. Ncw York: Academic Press, 1965.

ELSBERG, C. A., \& LEVY, I. The sense of smell: I. A new and simple method of quantitative olfactometry. Bulletin of the Neurological Institute of New York, 1935, 4, 5-19.

JONES, I. N. A test of the Elsberg technique of olfactometry. American Journal of Psychology, 1953, 66, 81-85.
OUGH, C. S., \& STONE, H. An olfactometer for rapid and critical odor measurement. Joumal of Food Sciences, 1961, 26, 452-456.

STONE, H. Physiological and psychological aspects of the olfactory response of man. In $\mathrm{N}$. Tanyolac (Ed.), Theories of odors and odor measurement. England: Technivision, 1968.

STONE, H. Some factors affecting olfactory sensitivity and odor intensity. Thesis. University of California at Davis, $112 \mathrm{pp} ., 1963 \mathrm{~b}$.

STONE, $H$. Techniques for odor measurement: Olfactometric vs. sniffing. Joumal of Food Sciences, 1963a, 2, 719-725.

STONE, H., \& BOSLEY, J. J. Olfactory discrimination and Weber's Law. Perceptual \& Motor Skills, 1965, 20, 657-665.

STONE, H., PANGBORN, R. M., \& OUGH, C. S. Techniques for sensory evaluation of food odors. Advanced Food Research, 1965, 14, 1-32.

STONE, H., PRYOR, G., \& COLWELL, J. Olfactory detection thresholds in man under conditions of rest and exercise. Perception \& Psychophysics, 1967, 2, 167-170.

STUIVER, M. Biophysics of the sense of smell. Thesis. Groningen University, The Netherlands, 99 pp., 1958.

WENZEL, B. M. Olfactometric method utilizing natural breathing in odor-free "environment." Science, 1955, 121, 802-803.

ZWAARDEMAKER, H. A camera inodorata. Perfumery Essential Oil Record, 1921, 12, 243-244.

\section{NOTES}

1. This research was supported in part by Grant No. NB 07832 from the Institute of Neurological Sciences of the National Institutes of Health to $\mathrm{H}$. Stone and in part by Grant No. 2002 FR-05522 from the Division of Facilities Resources. The authors acknowledge the advice and assistance of Mr. John S. Loram in design of the circuitry and of numerous staff members who participated as subjects.

2. Address: Life Sciences Division, Stanford Research Institute, Menlo Park, California 94025.

3. Stone, H., Pryor, G., \& Steinmetz, G. A comparison of two psychophysical techniques for the study of olfactory adaptation and recovery in man. Unpublished manuscript, 1969.

4. Stone, H., \& Pryor, G. Unpublished laboratory report, 1967.

\section{A simple tachistoscope for student labs}

\author{
RICHARD O. ROUSE and ROGER M. TARPY, WILLIAMS \\ COLLEGE, Williamstown, Mass. 01267
}

An inexpensive tachistoscope for student labs is described that utilizes a camera shutter to control exposure duration and a potentiometer to vary light intensity. Stimuli can be drawn or typed on $3 \times 5$ index cards.

There have been few notes on the construction of simple and inexpensive tachistoscopes. Most accounts concern special adaptations or designs that are too complicated for student lab use (Behr, 1960; Howard \& Evans, 1964). The present paper describes a simple tachistoscope that can be built inexpensively for use in student labs.

The basic component in this design is a camera shutter (llex Optical Company, Rochester, New York, No. 1 Universal, $\$ 28.00$ ) that is mounted on one wall of a wooden box directly opposite a $2 \times 4$-in. opening. The inside dimensions of the box are 6 in. high $\times 8$ in. wide $\times 10 \frac{1}{2}$ in. long. On the outside of the opening is a card holder made from grooved, wooden strips that center a standard $3 \times 5$-in. index card in the window (see Fig. 1). On the inside wall (placed $1 / 2$ in. from the shutter on three sides) are located three 3-V flashlight bulbs wired in parallel. The wires extend under the lid of the box to two flashlight battery brackets. With the inside of the box painted white, illumination from the bulbs is bright enough to offset any leakage from the card opening. Furthermore, it is possible to wire a small potentiometer (e.g., Clarostat, A 43-20, 93q) in series with the

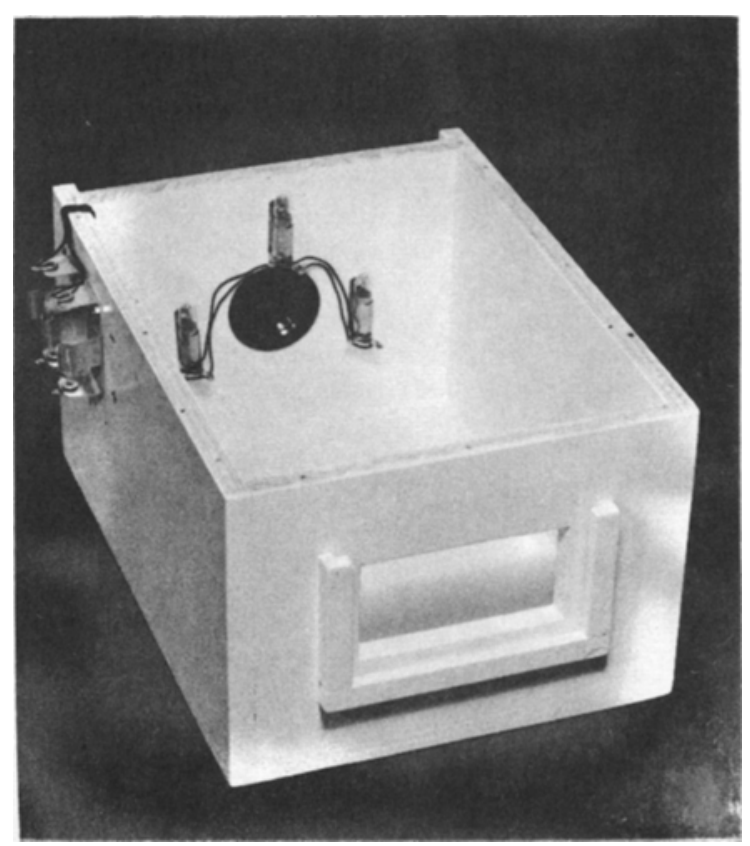

Fig. 1. Back view of the tachistoscope for student lab use (cover omitted). 